Notfall Rettungsmed 2015 · 18:649-650

DOI 10.1007/s10049-015-0111-z

Online publiziert: 13. November 2015

(c) Springer-Verlag Berlin Heidelberg 2015

CrossMark

\author{
B.W. Böttiger ${ }^{1,4} \cdot$ M. Baubin $^{2} \cdot$ R. Mauri ${ }^{3} \cdot$ B. Dirks ${ }^{1}$ \\ ${ }^{1}$ German Resuscitation Council, Ulm Deutschland \\ ${ }^{2}$ Austrian Resuscitation Council, Innsbruck, Österreich \\ ${ }^{3}$ Swiss Resuscitation Council, Bern, Schweiz \\ ${ }^{4}$ European Resuscitation Council, Niel, Belgien
}

\title{
Leitlinien zur Reanimation 2015 des European Resuscitation Council
}

Notfall+Rettungsmedizin publiziert in diesem Heft zum vierten Mal die autorisierte deutschsprachige Übersetzung der aktualisierten Leitlinien zur Reanimation des European Resuscitation Council (ERC) durch den Deutschen Rat für Wiederbelebung - German Resuscitation Council (GRC), den Österreichischen Rat für Wiederbelebung Austrian Resuscitation Council (ARC) und den Schweizer Rat für Wiederbelebung - Swiss Resuscitation Council (SRC).

In seinem Editorial „2015 Resuscitation Guidelines" ${ }^{\text {"1 }}$ beschreibt Dr. Jerry Nolan, der Co-chairman des ILCOR-Prozesses, nach welcher umfassenden, geradezu peniblen Auswertung der Literatur der wissenschaftliche Konsens für diese Leitlinien entstand. Basis für die Redaktionsgruppen (Writing Groups) der Reanimationsgesellschaften in aller Welt war dieser ILCOR „Consensus on Science With Treatment Recommendations“.

Danach konnten dann für Europa die Writing Groups des ERC daran gehen, diese wissenschaftliche Basis in aktualisierte Leitlinien für die Bereiche lebensrettende Basismaßnahmen, erweiterte Reanimationsmaßnahmen, Reanimation unter besonderen Bedingungen, Behandlung nach erfolgreicher Reanimation, lebensrettende Maßnahmen bei Kindern und Neugeborenen, das akute Koronarsyndrom sowie die speziellen Aspekte Ausbildung und Ethik umzusetzen. Diese Leitlinien geben die

1 Nolan J (2015) Resuscitation Guidelines, Notfall Rettungsmed. doi:10.1007/s10049-015-0102-0. weltweit akzeptierte und auf höchstmöglichem Evidenzniveau basierende Sicht wider, wie Reanimation wirkungsvoll und sicher durchgeführt werden kann. Dabei profitieren die Leitlinien von Kooperation. Schon 2010 waren die Autoren des Koronarkapitels auch bei den ESCLeitlinien beteiligt. So stimmen auch 2015 die Aussagen von ERC und ESC (European Society of Cardiology) überein. Jetzt hat sich die ERC Writing Group für die Leitlinien $\mathrm{zu}$ „Post Resuscitation Care" mit der European Society of Intensive Care Medicine zusammengetan und gemeinsame Leitlinien erarbeitet. Völlig neu sind die Bewertungen und Empfehlungen zur Ersten Hilfe. Hier hat die Writing Group „First Aid“ zwar noch viele Lücken in den wissenschaftlichen Grundlagen zu beklagen und berichtet ehrlicherweise, dass das Kapitel bei weitem nicht vollständig ist und vieles auf Expertenkonsens und gesundem Menschenverstand beruht. Dennoch ist dies ein erster wichtiger Schritt, um viele Meinungen und Traditionen auf diesem Feld auf den Prüfstand zu stellen und in den nächsten Jahren die wissenschaftliche Basis zu verbreitern.

Der ERC bekräftigt den 2010 gefundenen Kompromiss: „bei jeder Wiederbelebung soll eine Thoraxkompression durchgeführt werden. Notfallzeugen, die ausgebildet und in der Lage sind, eine Atemspende durchzuführen, sollen Herzdruckmassage und Atemspende kombinieren."

Um dieses Ziel „Herzdruckmassage durch jeden" $" z u$ erreichen, werden drei Aufgaben in den Vordergrund gestellt:
- Erkennen des Kreislaufstillstands: Noch immer wird bei Schnappatmung oder Krampfanfällen die Situation oft verkannt. Unsere Hoffnungen ruhen dabei auf dem Leitstellenpersonal in Europa. Der Disponent muss aus der Schilderung des Laien beim Notruf den Verdacht auf einen Kreislaufstillstand haben und die Überlebenskette starten.

- Die hohen Erwartungen, die wir 2010 in die „Telefonreanimation“ gelegt haben, haben sich wegen spärlicher Implementierung bisher leider nicht erfüllt. Auch hier wird der Disponent von den Leitlinien in die Pflicht genommen, er muss jeden Ersthelfer zur Thoraxkompression anleiten.

- Und noch eine weitere Schlüsselstellung haben Leitstellen bei der Reanimation: In Zukunft soll es selbstverständlich sein, dass sie in ihrem Einzugsbereich Übersicht über die Platzierung der AED haben und Ersthelfer, die nicht unmittelbar mit dem Wichtigsten - der Herzdruckmassage - beschäftigt sind, mit dem Gerät zum Patienten führen können.

Die Basisreanimation selbst hat keine wesentliche Änderung erfordert. Dies ist unter dem Aspekt der Kontinuität und Durchdringung sehr zu begrüßen. Es ist doch so einfach, man braucht nur zwei Hände. Wenn wir es nicht schaffen, die Laienreanimationshäufigkeit in Europa überall auf den hohen Stand der skandinavischen Länder zu bringen, wird der Überlebensfortschritt gering bleiben, denn ein wesentlich früheres Eintreffen 


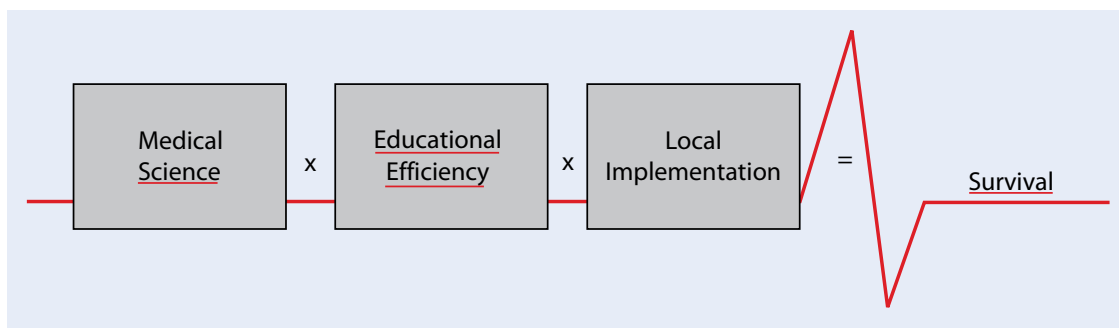

Abb. $1 \Delta$ Die Überlebensformel

des Rettungsdienstes ist wirtschaftlich nicht darstellbar. Deshalb müssen wir früh beginnen, nämlich bei den Schulkindern. Mit 12 Jahren sind sie körperlich dazu im Stande und viele Einzelfälle und etliche Studien zeigen: Sie trauen sich auch. Eine Doppelstunde pro Jahr ab der 7. Klasse ist ausreichend. Die Schüler können von dazu ausgebildeten Lehrern unterrichtet werden. Entsprechende Empfehlungen werden in Deutschland von der Kultusministerkonferenz, in Österreich von der Konferenz der Landtagspräsidenten seit 2014 und der Weltgesundheitsorganisation (WHO) seit 2015 unterstützt. Der GRC hat hierfür gemeinsam mit allen Hilfsorganisationen - ein Ausbildungscurriculum entwickelt (www.grc-org.de).

Die Leitlinien zu den „Erweiterten Maßnahmen" betonen den Einsatz der Kapnographie zur Kontrolle von Tubuslage, Reanimationsqualität und ROSC. Die Empfehlungen zur Medikation bleiben unverändert, sie stehen nicht mehr im Vordergrund und auch die Empfehlungen zum Airway-Management sind weitgehend unverändert. Erstmalig wird der Einsatz eines extrakorporalen Kreislaufs als Überbrückungsmaßnahme im Einzelfall in Betracht gezogen.

In Kliniken ist das frühe Erkennen und Beseitigen von Störungen der Vitalfunktionen, die zu einem Kreislaufstillstand führen können, bisher häufig nicht selbstverständlich - obgleich unsere Patienten sich eigentlich darauf verlassen. Die ALS-Leitlinien des ERC betonen daher erneut die Bedeutung innerklinischer Notfallteams („Rapid Response Team“, „Medical Emergency Team“) zur Versorgung des sich verschlechternden Patienten, damit der innerklinische Kreislaufstillstand vermieden wird.

Die Leitlinien zur Behandlung nach Reanimation bilden 2015 ein neues, eigenes
Kapitel. Die Überlebenschancen sind höher, wenn die Patienten - im Einzelfall sogar unter laufender Reanimation nach einem prähospitalen Kreislaufstillstand in spezielle Zentren (sog. „Cardiac Arrest Center") eingeliefert werden. Solche Zentren müssen jederzeit die Möglichkeit zur akuten Koronarintervention haben, eine entsprechend hohe Fallzahl aufweisen und ein gezieltes TemperaturManagement durchführen. Meist ist der Kreislaufstillstand ja die Folge eines akuten Herzinfarkts. Werden die für den Infarkt verantwortlichen Koronararterien innerhalb der ersten 1-2 $\mathrm{h}$ nach dem Ereignis dilatiert, dann verbessert dies deutlich die Prognose. Gleichzeitig enthalten die neuen Leitlinien überarbeitete Empfehlungen zum Temperaturmanagement. Nach Kreislaufstillstand sollen bewusstlose Patienten unabhängig vom initialen Herzrhythmus für mindestens 24 h auf $32-34$ oder $36^{\circ} \mathrm{C}$ gekühlt werden. Fieber muss ebenso wie eine Hyperoxie in jedem Fall für $72 \mathrm{~h}$ vermieden werden. Für die Prognostizierung - die jetzt frühestens nach $72 \mathrm{~h}$ empfohlen wird - gibt es ganz neue multimodale Vorgaben. Empfehlungen zur Rehabilitation nach überlebtem Kreislaufstillstand behandeln als neuer Abschnitt den systematischen Aufbau der Nachbetreuung; sie gehen auch auf zwar geringere, für die Lebensqulität des Patienten aber bedeutsame kognitive und emotionale Defizite ein.

Am Reanimationsablauf bei Kindern ändert sich wenig. Aber der grundsätzliche Umgang mit dem Problem, dass Kinderreanimationen - Gott sei Dank - selten sind und der Notfallzeuge dann sicher nicht mehr irgendwelche speziellen Maßnahmen für Kinder erinnert, ist weiter pragmatischer geworden. Das Prinzip ist: Wer nicht besondere Verantwortung für Kinder trägt (und sich deshalb auskennen muss), soll Kinder reanimieren wie Erwachsene.
Das Kap. 7 „Neugeborene“ stellt weniger die Reanimation als die Unterstützung des perinatalen Anpassungsprozesses in den Vordergrund; das Kind soll nicht reanimationspflichtig werden.

Alles in allem dürfen wir konstatieren: Die Leitlinien 2015 enthalten keine revolutionären Änderungen gegenüber 2010; sie sind eher eine Evolution mit wichtigen Änderungen im Detail, und das wird die Vermittlung erleichtern.

Wie 2010 möchten wir die Leserinnen und Leser unserer Zeitschrift an die „Überlebensformel“ erinnern: Überleben ist: Wissenschaft mal Ausbildung mal Implementierung (『Abb. 1)!

Es wird darauf ankommen, diese Leitlinien Laien, Leitstellen, professionellen Helfern und Politikern zu vermitteln und die Konzepte erfolgreich zu implementieren, an der Spitze das Prinzip „Jeder kann reanimieren - alles was man braucht sind zwei Hände"!

Ihre

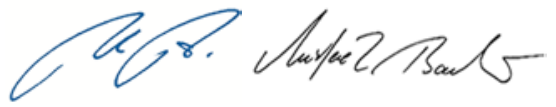

Bernd W. Böttiger Michael Baubin

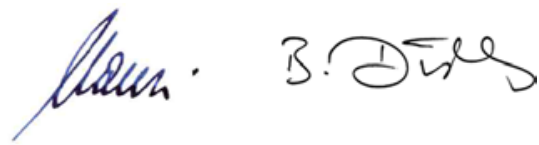

Romano Mauri Burkhard Dirks

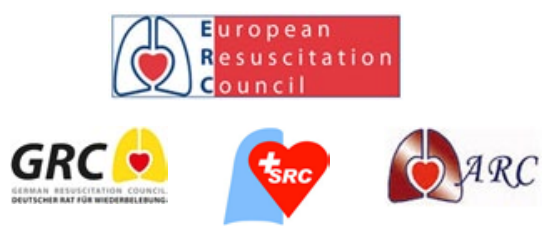

\section{Korrespondenzadresse}

Dr. rer. nat. Dr. med. B. Dirks

German Resuscitation Council

co. Universitätsklinikum Ulm co. Universitätsklinikum Ulm

Sektion Notfallmedizin, 89070 UIm

dirks@grc-org.de

Interessenkonflikt. B.W. Böttiger, M. Baubin, R. Mauri und B. Dirks geben an, dass kein Interessenkonflikt besteht. 